\title{
ON THE EFFECT OF SUBSTRATE HEATING ON THE RATE OF GROWTH OF EPITAXIAL LAYERS
}

\section{E. L. PANKRATOV}

Nizhny Novgorod State University

23 Gagarin Avenue

Nizhny Novgorod, 603950

Russia

Nizhny Novgorod State Technical University

24 Minin Street

Nizhny Novgorod, 603950

Russia

e-mail: elp2004@mail.ru

\begin{abstract}
In this paper, we estimate the rate of growth of epitaxial layers from the gas phase. We study dependence of the rate on the value of the heating of the substrate. By using the previously introduced approach of mass and heat transfer analysis, analytical dependencies of the considered rate on the parameters were obtained. In this paper, based on recently introduced approach, we analyzed mass and heat transport during growth of epitaxial layers in reactors for epitaxy from gas phase with sloping keeper with account native convection. Based on recently introduced approach, we estimate rate of growth of films and analyzed dependences of the rate on physical and technological parameters.
\end{abstract}

Keywords and phrases: growth from gas phase, changing of growth rate, prognosis of technological process.

Received April 30, 2019

(C) 2019 Scientific Advances Publishers 


\section{Introduction}

At the present time, different heterostructures are widely used to manufacture different devices of solid-state electronics. The most common methods for growing heterostructures are epitaxy from the gas and liquid phases, magnetron sputtering, molecular beam epitaxy. Manufacturing and using of heterostructures have been considered in large number of experimental works because of their wide using [1-11]. At the same time, a relatively small number of works are devoted to predicting the processes of epitaxy [12]. The main aim of the present paper is to analyze the growth of epitaxial layers with changes in the values of parameters of the growth process taking into account natural convection.

In this paper, we consider a reactor for epitaxy from the gas phase (see Figure 1). This reactor consists of a casing, a substrate holder with a substrate, and a helix around the casing in the region of formation of the epitaxial layer in order to provide induction heating for activating the chemical reactions that occur during the decomposition of reagents and the formation of an epitaxial layer. A gaseous mixture of reagents is supplied to the inlet of the reaction chamber together with the carrier gas. The main goal of this paper is to estimate the rate of growth of an epitaxial layer and to analyze its dependence on the substrate temperature.

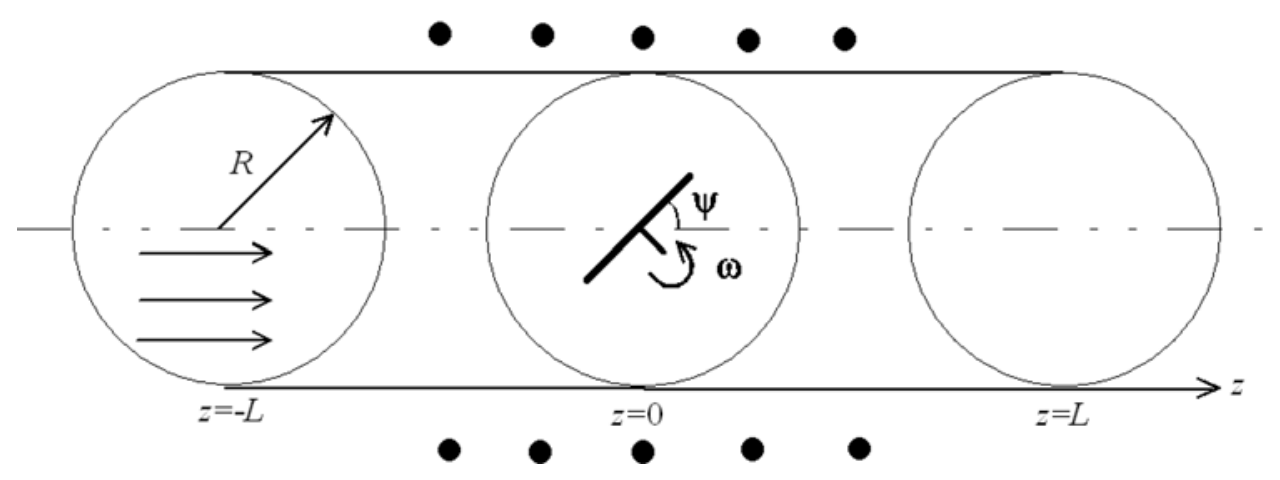

Figure 1(a). Structure of reactor for gas phase epitaxy with sloping keeper of substrate. 


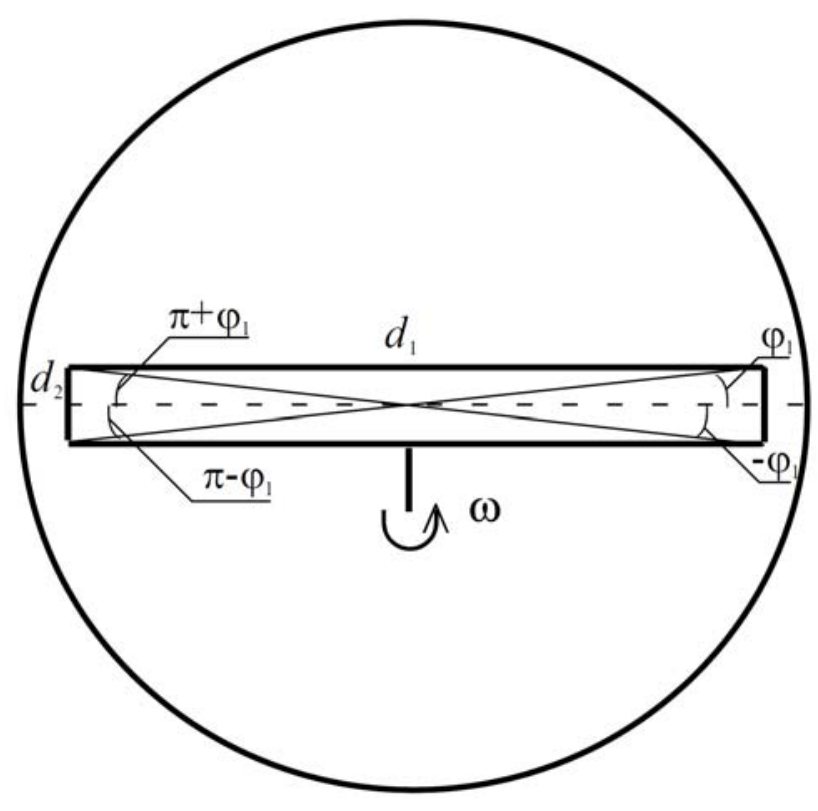

Figure 1(b). View from side of keeper of substrate and approximation of the keeper by sloping lines with angle of sloping $\varphi_{1}$.

\section{Method of Solution}

First of all we analyze spatio-temporal distribution of temperature. To analyze the distribution we determine the solution of the second Fourier law [13]

$$
\begin{aligned}
c \frac{\partial T(r, \varphi, z, t)}{\partial t}= & \operatorname{div}\{\lambda \cdot \operatorname{grad}[T(r, \varphi, z, t)] \\
& -\vec{v}(r, \varphi, z, t) \cdot c(T) \cdot T(r, \varphi, z, t) \cdot C(r, \varphi, z, t)\}+p(r, \varphi, z, t),
\end{aligned}
$$

where $\vec{v}$ is the speed of flow of mixture of gases-reagents (we consider gases-reagents as ideal gases); $c$ is the heat capacity; $T(r, \varphi, z, t)$ is the spatio-temporal distribution of temperature; $p(r, \varphi, z, t)$ is the density of power in the system substrate keeper of substrate; $r, \varphi, z$, and $t$ are the cylindrical coordinates and time; $C(r, \varphi, z, t)$ is the spatio-temporal 
distribution of concentration of mixture of gases-reagents; and $\lambda$ is the heat conductivity. Value of heat conductivity could be determine by the following relation: $\lambda=\bar{\nu} \bar{l} c_{\nu} \rho / 3$, where $\bar{\nu}$ is the speed of the gas molecules, $\bar{l}$ is the average free path of gas molecules between collisions, $c_{\nu}$ is the specific heat at constant volume, and $\rho$ is the density of gas.

To solve the Equation (1), we shall to take into account moving of mixture of gases and concentration of the mixture. We determine speed of the moving and the concentration by solving the equation of NavierStokes and the second Fick's law, respectively. We also assume that radius of keeper of substrate $R$ essentially larger, than thickness of diffusion and near-boundary layers. We also assume, that stream of gas is laminar. In this situation, the appropriate equations could be written as

$$
\begin{gathered}
\frac{\partial \vec{v}}{\partial t}+(\vec{\nu} \cdot \nabla) \vec{\nu}=-\nabla\left(\frac{P}{\rho}\right)+\nu \Delta \vec{\nu}, \\
\frac{\partial C(r, \varphi, z, t)}{\partial t}=\operatorname{div}\{D \cdot \operatorname{grad}[C(r, \varphi, z, t)]-\vec{\nu}(r, \varphi, z, t) \cdot C(r, \varphi, z, t)\},
\end{gathered}
$$

where $D$ is the diffusion coefficient of mixture of gases-reagents; $P$ is the pressure; $\rho$ is the density; and $\nu$ is the kinematic viscosity. Let us consider the regime of the limiting flow, when all forthcoming to the disk molecules of deposit material are deposing on the substrate, flow is homogeneous and one dimension. In this case boundary and initial conditions could be written as

$$
\begin{aligned}
& C(r, \varphi,-L, t)=C_{0}, C\left(r,-\varphi_{1}, z, t\right)=C\left(r, \varphi_{1}, z, t\right)=C\left(r, \pi-\varphi_{1}, z, t\right)=C \\
& \left(r, \pi+\varphi_{1}, z, t\right), C(r, \varphi, z, 0)=C_{0} \delta(z+L), C(0, \varphi, z, t) \neq \infty,\left.C(r, \varphi, z, t)\right|_{s}=0, \\
& T\left(r,-\varphi_{1}, z, t\right)=T\left(r, \varphi_{1}, z, t\right)=T\left(r, \pi-\varphi_{1}, z, t\right)=T\left(r, \pi+\varphi_{1}, z, t\right), \\
& \left.\frac{\partial C(r, \varphi, z, t)}{\partial r}\right|_{r=R}=0,-\left.\lambda \frac{\partial T(r, \varphi, z, t)}{\partial r}\right|_{S}=\sigma T^{4}(R, \varphi, z, t), T(r, \varphi, z, 0)=T_{r}, \\
& \left.\frac{\partial T(r, \varphi, z, t)}{\partial \varphi}\right|_{\varphi=0}=\left.\frac{\partial T(r, \varphi, z, t)}{\partial \varphi}\right|_{\varphi=2 \pi},-\left.\lambda \frac{\partial T(r, \varphi, z, t)}{\partial z}\right|_{S}=\sigma T^{4}(r, \varphi,-L, t),
\end{aligned}
$$


$T(0, \varphi, z, t) \neq \infty,\left.\frac{\partial \nu_{r}(r, \varphi, z, t)}{\partial r}\right|_{r=0}=0,\left.\frac{\partial \nu_{r}(r, \varphi, z, t)}{\partial r}\right|_{r=R}=0$

$\nu_{r}\left(r,-\varphi_{1}, z, t\right)=\nu_{r}\left(r, \varphi_{1}, z, t\right)=\nu_{r}\left(r, \pi-\varphi_{1}, z, t\right)=\nu_{r}\left(r, \pi+\varphi_{1}, z, t\right)$,

$\nu_{\varphi}\left(r,-\varphi_{1}, z, t\right)=v_{\varphi}\left(r, \varphi_{1}, z, t\right)=v_{\varphi}\left(r, \pi-\varphi_{1}, z, t\right)=v_{\varphi}\left(r, \pi+\varphi_{1}, z, t\right)$,

$\nu_{z}\left(r,-\varphi_{1}, z, t\right)=\nu_{z}\left(r, \varphi_{1}, z, t\right)=\nu_{z}\left(r, \pi-\varphi_{1}, z, t\right)=\nu_{z}\left(r, \pi+\varphi_{1}, z, t\right)$,

$\nu_{r}(r, \varphi,-L, t)=0, \nu_{r}(r, \varphi, L, t)=0, \nu_{r}(0, \varphi, z, t) \neq \infty, \nu_{z}(r, \varphi,-L, t)=V_{0}$,

$\nu_{z}\left(r \pm d_{2} / 2, \varphi, z \in\left[-d_{2} / 2, d_{2} / 2\right], 0\right)=\omega \cdot z \cdot \cos \psi \operatorname{tg}\left(\varphi_{1}\right)$,

$\nu_{\varphi}(r, \varphi, L, t)=0, \nu_{\varphi}(0, \varphi, z, t) \neq \infty, v_{z}(r, \varphi, 0, t)=0, \nu_{z}(r, \varphi, L, t)=V_{0}$,

$\nu_{z}(0, \varphi, z, t) \neq \infty, \nu_{r}(r, \varphi, z, 0)=0, \nu_{\varphi}(r, \varphi, z, 0)=0$,

where $\sigma=5.67 \cdot 10^{-8} \mathrm{~W} \cdot \mathrm{m}^{-2} \cdot \mathrm{K}^{-4}, T_{r}$ is the room temperature, $\omega$ is the frequency of rotation of the substrate.

Equations for components of velocity of flow with account cylindrical system of coordinate could be written as

$$
\begin{gathered}
\frac{\partial v_{r}}{\partial t}=\nu\left\{\frac{1}{r} \frac{\partial}{\partial r}\left[r \frac{\partial v_{r}(r, \varphi, z, t)}{\partial r}\right]+\frac{1}{r^{2}} \frac{\partial^{2} v_{r}(r, \varphi, z, t)}{\partial \varphi^{2}}+\frac{\partial^{2} v_{r}(r, \varphi, z, t)}{\partial z^{2}}\right\} \\
-v_{r} \frac{\partial v_{r}}{\partial r}-\frac{v_{\varphi}}{r} \frac{\partial v_{\varphi}}{\partial \varphi}-v_{z} \frac{\partial v_{z}}{\partial z}-\frac{\partial}{\partial r}\left(\frac{P}{\rho}\right) \\
\frac{\partial v_{\varphi}}{\partial t}=\nu\left\{\frac{1}{r} \frac{\partial}{\partial r}\left[r \frac{\partial v_{\varphi}(r, \varphi, z, t)}{\partial r}\right]+\frac{1}{r^{2}} \frac{\partial^{2} v_{\varphi}(r, \varphi, z, t)}{\partial \varphi^{2}}+\frac{\partial^{2} v_{\varphi}(r, \varphi, z, t)}{\partial z^{2}}\right\} \\
-v_{r} \frac{\partial v_{r}}{\partial r}-\frac{v_{\varphi}}{r} \frac{\partial v_{\varphi}}{\partial \varphi}-v_{z} \frac{\partial v_{z}}{\partial z}-\frac{1}{r} \frac{\partial}{\partial \varphi}\left(\frac{P}{\rho}\right)
\end{gathered}
$$




$$
\begin{gathered}
\frac{\partial v_{z}}{\partial t}=v\left\{\frac{1}{r} \frac{\partial}{\partial r}\left[r \frac{\partial v_{z}(r, \varphi, z, t)}{\partial r}\right]+\frac{1}{r^{2}} \frac{\partial^{2} v_{z}(r, \varphi, z, t)}{\partial \varphi^{2}}+\frac{\partial^{2} v_{z}(r, \varphi, z, t)}{\partial z^{2}}\right\} \\
-v_{r} \frac{\partial v_{r}}{\partial r}-\frac{v_{\varphi}}{r} \frac{\partial v_{\varphi}}{\partial \varphi}-v_{z} \frac{\partial v_{z}}{\partial z}-\frac{\partial}{\partial z}\left(\frac{P}{\rho}\right) .
\end{gathered}
$$

We determine solution of this system of equations by using of method of averaging of function corrections [14-19]. Framework this approach first of all we determine the first-order approximation of components of speed of flow of mixture of gases. To determine the first-order approximation, we replace of the required functions on their average values $\nu_{r} \rightarrow \alpha_{1 r}, \nu_{\varphi} \rightarrow \alpha_{1 \varphi}, \nu_{z} \rightarrow \alpha_{1 z}$ in the right sides of equations of system (5). After the replacement and calculation required derivatives, we obtain equations for the first-order approximations of the components

$$
\frac{\partial \nu_{1 r}}{\partial t}=-\frac{\partial}{\partial r}\left(\frac{P}{\rho}\right), \quad \frac{\partial \nu_{1 \varphi}}{\partial t}=-\frac{1}{r} \frac{\partial}{\partial \varphi}\left(\frac{P}{\rho}\right), \quad \frac{\partial \nu_{1 z}}{\partial t}=-\frac{\partial}{\partial z}\left(\frac{P}{\rho}\right) .
$$

Integration of the left and the right sides on time of the relations (6) gives us possibility to obtain the first-order approximations of the components of speed of flow in the final form

$$
\nu_{1 r}=-\frac{\partial}{\partial r} \int_{0}^{t} \frac{P}{\rho} d \tau, \quad \nu_{1 \varphi}=-\frac{1}{r} \frac{\partial}{\partial \varphi} \int_{0}^{t} \frac{P}{\rho} d \tau, \quad \nu_{1 z}=-\frac{\partial}{\partial z} \int_{0}^{t} \frac{P}{\rho} d \tau .
$$

The second-order approximations of components of speed of flow could be obtain by replacement of the required functions on the following sums: $\nu_{r} \rightarrow \alpha_{2 r}+\nu_{1 r}, \nu_{\varphi} \rightarrow \alpha_{2 \varphi}+\nu_{2 r}, \nu_{z} \rightarrow \alpha_{2 z}+\nu_{2 r}$. The average values $\alpha_{2 r}, \alpha_{2 \varphi}, \alpha_{2 z}$ are not yet known. Approximations for the components could be written as

$$
\begin{aligned}
\frac{\partial \nu_{2 r}}{\partial t}= & \nu\left[\frac{1}{r} \frac{\partial}{\partial r}\left(r \frac{\partial \nu_{1 r}}{\partial r}\right)+\frac{1}{r^{2}} \frac{\partial^{2} \nu_{1 r}}{\partial \varphi^{2}}+\frac{\partial^{2} \nu_{1 r}}{\partial z^{2}}\right]-\frac{\partial}{\partial r}\left(\frac{P}{\rho}\right) \\
& -\left(\alpha_{2 r}+\nu_{1 r}\right) \frac{\partial \nu_{1 r}}{\partial r}-\frac{\left(\alpha_{2 \varphi}+\nu_{1 \varphi}\right)}{r} \frac{\partial \nu_{1 r}}{\partial \varphi}-\left(\alpha_{2 z}+\nu_{1 z}\right) \frac{\partial \nu_{1 r}}{\partial z}
\end{aligned}
$$




$$
\begin{aligned}
\frac{\partial \nu_{2 \varphi}}{\partial t}= & \nu\left[\frac{1}{r} \frac{\partial}{\partial r}\left(r \frac{\partial \nu_{1 \varphi}}{\partial r}\right)+\frac{1}{r^{2}} \frac{\partial^{2} \nu_{1 \varphi}}{\partial \varphi^{2}}+\frac{\partial^{2} \nu_{1 \varphi}}{\partial z^{2}}\right]-\frac{1}{r} \frac{\partial}{\partial \varphi}\left(\frac{P}{\rho}\right) \\
& -\left(\alpha_{2 r}+\nu_{1 r}\right) \frac{\partial \nu_{1 \varphi}}{\partial r}-\frac{\left(\alpha_{2 \varphi}+\nu_{1 \varphi}\right)}{r} \frac{\partial \nu_{1 \varphi}}{\partial \varphi}-\left(\alpha_{2 z}+\nu_{1 z}\right) \frac{\partial \nu_{1 \varphi}}{\partial z}, \\
\frac{\partial \nu_{2 z}}{\partial t}= & \nu\left[\frac{1}{r} \frac{\partial}{\partial r}\left(r \frac{\partial \nu_{1 z}}{\partial r}\right)+\frac{1}{r^{2}} \frac{\partial^{2} \nu_{1 z}}{\partial \varphi^{2}}+\frac{\partial^{2} \nu_{1 z}}{\partial z^{2}}\right]-\frac{\partial}{\partial z}\left(\frac{P}{\rho}\right) \\
& -\left(\alpha_{2 r}+\nu_{1 r}\right) \frac{\partial \nu_{1 z}}{\partial r}-\frac{\left(\alpha_{2 \varphi}+\nu_{1 \varphi}\right)}{r} \frac{\partial \nu_{1 z}}{\partial \varphi}-\left(\alpha_{2 z}+v_{1 z}\right) \frac{\partial \nu_{1 z}}{\partial z}
\end{aligned}
$$

Integration of the above equations on time leads to the following result:

$$
\begin{aligned}
\nu_{2 r}= & \nu \int_{0}^{t}\left[\frac{1}{r} \frac{\partial}{\partial r}\left(r \frac{\partial \nu_{1 r}}{\partial r}\right)+\frac{1}{r^{2}} \frac{\partial^{2} \nu_{1 r}}{\partial \varphi^{2}}+\frac{\partial^{2} \nu_{1 r}}{\partial z^{2}}\right] d \tau-\frac{\partial}{\partial r}\left(\int_{0}^{t} \frac{P}{\rho} d \tau\right) \\
& -\int_{0}^{t}\left(\alpha_{2 r}+\nu_{1 r}\right) \frac{\partial \nu_{1 r}}{\partial r} d \tau-\int_{0}^{t} \frac{\left(\alpha_{2 \varphi}+\nu_{1 \varphi}\right)}{r} \frac{\partial \nu_{1 r}}{\partial \varphi} d \tau-\int_{0}^{t}\left(\alpha_{2 z}+\nu_{1 z}\right) \frac{\partial \nu_{1 r}}{\partial z} d \tau, \\
\nu_{2 \varphi}= & \nu \int_{0}^{t}\left[\frac{1}{r} \frac{\partial}{\partial r}\left(r \frac{\partial \nu_{1 \varphi}}{\partial r}\right)+\frac{1}{r^{2}} \frac{\partial^{2} \nu_{1 \varphi}}{\partial \varphi^{2}}+\frac{\partial^{2} \nu_{1 \varphi}}{\partial z^{2}}\right] d \tau-\frac{1}{r} \frac{\partial}{\partial \varphi}\left(\int_{0}^{t} \frac{P}{\rho} d \tau\right) \\
& -\int_{0}^{t}\left(\alpha_{2 r}+\nu_{1 r}\right) \frac{\partial \nu_{1 \varphi}}{\partial r} d \tau-\int_{0}^{t} \frac{\left(\alpha_{2 \varphi}+\nu_{1 \varphi}\right)}{r} \frac{\partial \nu_{1 \varphi}}{\partial \varphi} d \tau-\int_{0}^{t}\left(\alpha_{2 z}+\nu_{1 z}\right) \frac{\partial \nu_{1 \varphi}}{\partial z} d \tau, \\
\nu_{2 z}= & V_{0}+\nu \int_{0}^{t}\left[\frac{1}{r} \frac{\partial}{\partial r}\left(r \frac{\partial \nu_{1 z}}{\partial r}\right)+\frac{1}{r^{2}} \frac{\partial^{2} \nu_{1 z}}{\partial \varphi^{2}}+\frac{\partial^{2} \nu_{1 z}}{\partial z^{2}}\right] d \tau-\frac{\partial}{\partial z}\left(\int_{0}^{t} \frac{P}{\rho} d \tau\right) \\
& -\int_{0}^{t}\left(\alpha_{2 r}+\nu_{1 r}\right) \frac{\partial \nu_{1 z}}{\partial r} d \tau-\int_{0}^{t} \frac{\left(\alpha_{2 \varphi}+\nu_{1 \varphi}\right)}{r} \frac{\partial \nu_{1 z}}{\partial \varphi} d \tau-\int_{0}^{t}\left(\alpha_{2 z}+\nu_{1 z}\right) \frac{\partial \nu_{1 z}}{\partial z} d \tau .
\end{aligned}
$$


Further we determine the average values $\alpha_{2 r}, \alpha_{2 \varphi}, \alpha_{2 z}$. The average values have been calculated by the following relations [14-19]:

$$
\begin{aligned}
& \alpha_{2 r}=\frac{1}{\pi \Theta R^{2} L} \int_{0}^{\Theta} \int_{0}^{R} r \int_{0}^{2 \pi} \int_{-L}^{L}\left(\nu_{2 r}-v_{1 r}\right) d z d \varphi d r d t, \\
& \alpha_{2 \varphi}=\frac{1}{\pi \Theta R^{2} L} \int_{0}^{\Theta} \int_{0}^{R} r \int_{0}^{2 \pi} \int_{-L}^{L}\left(\nu_{2 \varphi}-v_{1 \varphi}\right) d z d \varphi d r d t \\
& \alpha_{2 z}=\frac{1}{\pi \Theta R^{2} L} \int_{0}^{\Theta} \int_{0}^{R} r \int_{0}^{2 \pi} \int_{-L}^{L}\left(\nu_{2 z}-\nu_{1 z}\right) d z d \varphi d r d t,
\end{aligned}
$$

where $\Theta$ is the continuance of moving of mixture of gases through considered horizontal reactor. Substitution of the first- and the secondorder approximations of the required components of speed into the relation (9) give us possibility to obtain system of equations to determine required average values

$$
\left\{\begin{array}{l}
A_{1} \alpha_{2 r}+B_{1} \alpha_{2 \varphi}+C_{1} \alpha_{2 z}=D_{1} \\
A_{2} \alpha_{2 r}+B_{2} \alpha_{2 \varphi}+C_{2} \alpha_{2 z}=D_{2} \\
A_{3} \alpha_{2 r}+B_{3} \alpha_{2 \varphi}+C_{3} \alpha_{2 z}=D_{3}
\end{array}\right.
$$

where

$$
\begin{aligned}
& A_{1}=1+\int_{0}^{\Theta}(\Theta-t) \int_{0}^{R} r \int_{0}^{2 \pi} \int_{-L}^{L} \frac{\partial \nu_{1 r}}{\partial r} d z d \varphi d r d t, B_{1}=\int_{0}^{\Theta}(\Theta-t) \int_{0}^{R} \int_{0}^{2 \pi} \int_{-L}^{L} \frac{\partial \nu_{1 r}}{\partial \varphi} d z d \varphi d r d t \\
& C_{1}=C_{2}=\frac{\pi}{2} \Theta^{2} R^{2} V_{0}, D_{1}=\nu \int_{0}^{\Theta} \int_{0}^{R} r \int_{0}^{2 \pi} \int_{-L}^{L}\left[\frac{1}{r} \frac{\partial}{\partial r}\left(r \frac{\partial \nu_{1 r}}{\partial r}\right)+\frac{1}{r^{2}} \frac{\partial^{2} \nu_{1 r}}{\partial \varphi^{2}}+\frac{\partial^{2} \nu_{1 r}}{\partial z^{2}}\right] \\
& d z d \varphi d r(\Theta-t) d t-\frac{\pi}{8} \Theta^{2} R^{2} V_{0}^{2}-\int_{0}^{\Theta}(\Theta-t) \int_{0}^{R} r \int_{0}^{2 \pi} \int_{-L}^{L} \nu_{1 r} \frac{\partial \nu_{1 r}}{\partial r} d z d \varphi d r d t
\end{aligned}
$$




$$
\begin{aligned}
& -\int_{0}^{\Theta} \int_{0}^{R} \int_{0}^{2 \pi} \int_{-L}^{L} \nu_{1 \varphi} \frac{\partial \nu_{1 r}}{\partial \varphi} d z d \varphi d r(\Theta-t) d t, A_{2}=\int_{0}^{\Theta}(\Theta-t) \int_{0}^{R} r \int_{0}^{2 \pi} \int_{-L}^{L} \frac{\partial \nu_{1 r}}{\partial r} d z d \varphi d r d t \\
& B_{2}=1+\int_{0}^{\Theta} \int_{0}^{R} \int_{0}^{2 \pi} \int_{-L}^{L} \frac{\partial \nu_{1}}{\partial \varphi} d z d \varphi d r(\Theta-t) d t, D_{2}=\nu \int_{0}^{\Theta}(\Theta-t) \int_{0}^{R} r \int_{0}^{2 \pi} \int_{-L}^{L}\left[\frac{1}{r} \frac{\partial}{\partial r}\left(r \frac{\partial \nu_{1 \varphi}}{\partial r}\right)\right. \\
& \left.+\frac{1}{r^{2}} \frac{\partial^{2} \nu_{1 \varphi}}{\partial \varphi^{2}}+\frac{\partial^{2} \nu_{1 \varphi}}{\partial z^{2}}\right] d z d \varphi d r d t-\int_{0}^{\Theta}(\Theta-t) \int_{0}^{R} r \int_{0}^{2 \pi} \int_{-L}^{L} \nu_{1 r} \frac{\partial \nu_{1 r}}{\partial r} d z d \varphi d r d t \\
& -\int_{0}^{\Theta}(\Theta-t) \int_{0}^{R} \int_{0}^{2 \pi} \int_{-L}^{L} \nu_{1 \varphi} \frac{\partial \nu_{1 r}}{\partial \varphi} d z d \varphi d r d t-\frac{\pi}{8} \Theta^{2} R^{2} V_{0}^{2}, C_{3}=1+\frac{\pi}{2} \Theta^{2} R^{2} V_{0}, \\
& A_{3}=\int_{0}^{\Theta}(\Theta-t) \int_{0}^{R} r \int_{0}^{2 \pi} \int_{-L}^{L} \frac{\partial \nu_{1 z}}{\partial r} d z d \varphi d r d t, B_{3}=\int_{0}^{\Theta}(\Theta-t) \int_{0}^{R} r \int_{0}^{2 \pi} \int_{-L}^{L} \frac{\partial \nu_{1 z}}{\partial \varphi} d z d \varphi d r d t \\
& D_{3}=\nu \int_{0}^{\Theta}(\Theta-t) \int_{0}^{R} r \int_{0}^{2 \pi} \int_{-L}^{L}\left[\frac{1}{r} \frac{\partial}{\partial r}\left(r \frac{\partial \nu_{1 z}}{\partial r}\right)+\frac{1}{r^{2}} \frac{\partial^{2} \nu_{1 z}}{\partial \varphi^{2}}+\frac{\partial^{2} \nu_{1 z}}{\partial z^{2}}\right] d z d \varphi d r d t \\
& -\frac{\pi}{8} \Theta^{2} R^{2} V_{0}^{2}-\int_{0}^{\Theta}(\Theta-t) \int_{0}^{R} r \int_{0}^{2 \pi} \int_{-L}^{L} \nu_{1 r} \frac{\partial \nu_{1} z}{\partial r} d z d \varphi d r d t-\int_{0}^{\Theta}(\Theta-t) \\
& \times \int_{0}^{R} r \int_{0}^{2 \pi} \int_{-L}^{L} \nu_{1 \varphi} \frac{\partial \nu_{1 z}}{\partial \varphi} d z d \varphi d r d t
\end{aligned}
$$

Solution of the above system of equations could be determined by standard approaches [20] and could be written as

$$
\alpha_{2 r}=\Delta_{r} / \Delta, \alpha_{2 \varphi}=\Delta_{\varphi} / \Delta, \alpha_{2 z}=\Delta_{z} / \Delta,
$$

where

$$
\begin{gathered}
\Delta=A_{1}\left(B_{2} C_{3}-B_{3} C_{2}\right)-B_{1}\left(A_{2} C_{3}-A_{3} C_{2}\right)+C_{1}\left(A_{2} B_{3}-A_{3} B_{2}\right), \\
\Delta_{r}=D_{1}\left(B_{2} C_{3}-B_{3} C_{2}\right)-B_{1}\left(D_{2} C_{3}-D_{3} C_{2}\right)+C_{1}\left(D_{2} B_{3}-D_{3} B_{2}\right),
\end{gathered}
$$




$$
\begin{aligned}
& \Delta_{\varphi}=D_{1}\left(B_{2} C_{3}-B_{3} C_{2}\right)-B_{1}\left(D_{2} C_{3}-D_{3} C_{2}\right)+C_{1} \times\left(D_{2} B_{3}-D_{3} B_{2}\right), \\
& \Delta_{z}=A_{1}\left(B_{2} D_{3}-B_{3} D_{2}\right)-B_{1}\left(A_{2} D_{3}-A_{3} D_{2}\right)+D_{1}\left(A_{2} B_{3}-A_{3} B_{2}\right) .
\end{aligned}
$$

In this section, we obtained components of velocity of stream of mixture of materials in gas phase, which are used for growth of heterostructure, and gas-carrier in the second-order approximation framework method of averaging of function corrections. Usually, the second-order approximation is enough good approximation to make qualitative analysis of the obtained solution and to obtain some quantitative results.

Let us rewrite Equations (1) and (3) by using cylindrical system of coordinate

$$
\begin{gathered}
c \frac{\partial T(r, \varphi, z, t)}{\partial t}=\lambda \frac{\partial^{2} T(r, \varphi, z, t)}{\partial r^{2}}+\frac{\lambda}{r^{2}} \frac{\partial^{2} T(r, \varphi, z, t)}{\partial \varphi^{2}}+\lambda \frac{\partial^{2} T(r, \varphi, z, t)}{\partial z^{2}} \\
-c \cdot \frac{\partial}{\partial r}\left[\nu_{r}(r, \varphi, z, t) \cdot C(r, \varphi, z, t) \cdot T(r, \varphi, z, t)\right]-\frac{c}{r} \frac{\partial}{\partial \varphi}\left[\nu_{\varphi}(r, \varphi, z, t) \cdot C\right. \\
(r, \varphi, z, t) \cdot T(r, \varphi, z, t)]-c \cdot \frac{\partial}{\partial z}\left[\nu_{z}(r, \varphi, z, t) \cdot C(r, \varphi, z, t) \cdot T(r, \varphi, z, t)\right] \\
+p(r, \varphi, z, t),
\end{gathered}
$$

$$
\begin{gathered}
\frac{\partial C(r, \varphi, z, t)}{\partial t}=\frac{1}{r} \frac{\partial}{\partial r}\left[r D \frac{\partial C(r, \varphi, z, t)}{\partial r}\right]-\frac{1}{r} \frac{\partial}{\partial r}\left[r C(r, \varphi, z, t) \nu_{r}(r, \varphi, z, t)\right] \\
+\frac{1}{r^{2}} \frac{\partial}{\partial \varphi}\left[D \frac{\partial C(r, \varphi, z, t)}{\partial \varphi}\right]-\frac{1}{r} \frac{\partial}{\partial \varphi}\left[C(r, \varphi, z, t) \nu_{\varphi}(r, \varphi, z, t)\right] \\
+\frac{\partial}{\partial z}\left[D \frac{\partial C(r, \varphi, z, t)}{\partial z}\right]-\frac{\partial}{\partial z}\left[C(r, \varphi, z, t) \nu_{z}(r, \varphi, z, t)\right] .
\end{gathered}
$$

In this section, we calculate components of speed of gas-reagents, which are used for growth of an epitaxial layer, and gas-carrier as the second-order approximations framework method of averaging of function corrections. Usually, the second-order approximation is enough good 
approximation to make qualitative analysis and to obtain some quantitative results. Results of analytical calculation have been checked by comparison with results of numerical simulation.

To determine spatio-temporal distributions of temperature and concentration of gas mixture we used method of average of function corrections. To determine the first-order approximations of the required functions, we replace them on their not yet known average values $\alpha_{1 T}$ and $\alpha_{1 C}$ in right sides of the above equations. Further, we used recently considered algorithm to obtain the first-order approximations of temperature and concentration of gas-reagents

$$
\begin{aligned}
T_{1}(r, \varphi, z, t)= & T_{r}+\int_{0}^{t} \frac{p(r, \varphi, z, \tau)}{c} d \tau-\alpha_{1 T} \alpha_{1 C} \int_{0}^{t} \frac{\partial \nu_{r}(r, \varphi, z, \tau)}{\partial r} d \tau \\
& -\frac{\alpha_{1 T} \alpha_{1 C}}{r} \int_{0}^{t} \frac{\partial \nu_{\varphi}(r, \varphi, z, \tau)}{\partial \varphi} d \tau-\alpha_{1 T} \alpha_{1 C} \int_{0}^{t} \frac{\partial \nu_{z}(r, \varphi, z, \tau)}{\partial z} d \tau \\
C_{1}(r, \varphi, z, t)= & -\alpha_{1 T} \alpha_{1 C} \int_{0}^{t} \frac{\partial \nu_{r}(r, \varphi, z, \tau)}{\partial r} d \tau-\frac{\alpha_{1 C}}{r} \int_{0}^{t} \frac{\partial\left[r v_{r}(r, \varphi, z, \tau)\right]}{\partial r} d \tau \\
& -\frac{\alpha_{1 C}}{r} \int_{0}^{t} \frac{\partial v_{\varphi}(r, \varphi, z, \tau)}{\partial \varphi} d \tau-\alpha_{1 C} \int_{0}^{t} \frac{\partial \nu_{z}(r, \varphi, z, \tau)}{\partial z} d \tau
\end{aligned}
$$

The above not yet known average values could be determined by the standard relations

$$
\begin{aligned}
& \alpha_{1 T}=\frac{1}{\pi \Theta R^{2} L} \int_{0}^{\Theta} \int_{0}^{R} r \int_{0}^{2 \pi} \int_{-L}^{L} T_{1}(r, \varphi, z, \tau) d z d \varphi d r d t, \\
& \alpha_{1 C}=\frac{1}{\pi \Theta R^{2} L} \int_{0}^{\Theta} \int_{0}^{R} r \int_{0}^{2 \pi} \int_{-L}^{L} C_{1}(r, \varphi, z, \tau) d z d \varphi d r d t .
\end{aligned}
$$


Substitution of the first-order approximations of temperature and concentration of gas mixter into relations (16) gives us the following results [20]:

$$
\begin{gathered}
\alpha_{1 C}=C_{0} / L \cdot\left[1+\frac{1}{\pi \Theta R L} \int_{0}^{\Theta}(\Theta-t) \int_{0}^{2 \pi} \int_{-L}^{L} \nu_{r}(R, \varphi, z, t) d z d \varphi d t+\frac{\Theta V_{0}}{R L}\right], \\
\alpha_{1 T}=\left[T_{r}+\frac{1}{\pi \Theta R^{2} L} \int_{0}^{\Theta}(\Theta-t) \int_{0}^{R} r \int_{0}^{2 \pi} \int_{-L}^{L} \frac{p(r, \varphi, z, t)}{c} d z d \varphi d r d t\right]\left\{1+\frac{C_{0}}{\pi \Theta R L^{2}}\left[\int_{0}^{\Theta}(\Theta-t)\right.\right. \\
\left.\times \int_{0}^{2 \pi} \int_{-L}^{L} \nu_{r}(R, \varphi, z, \tau) d z d \varphi d t-\frac{1}{\pi \Theta R^{2}} \int_{0}^{\Theta}(\Theta-t) \int_{0}^{R} \int_{0}^{2 \pi} \int_{-L}^{L} \nu_{r}(r, \varphi, z, \tau) d z d \varphi d r d t+\frac{V_{0}}{2}\right] \\
\left.\times\left[1+\frac{1}{\pi \Theta R L} \int_{0}^{\Theta}(\Theta-t) \int_{0}^{2 \pi} \int_{-L}^{L} \nu_{r}(R, \varphi, z, t) d z d \varphi d t+\frac{\Theta V_{0}}{R L}\right]^{-1}\right\}
\end{gathered}
$$

The second-order approximations of temperature and concentration of gases-reagents we determine framework the method of averaging of function corrections [16-21], i.e., by replacement of the required functions in right sides of Equations (12) and (13) on the following sums $T \rightarrow \alpha_{2 T}+T_{1}, C \rightarrow \alpha_{2 C}+C_{1}$. In this case, the second-order approximations of the above required functions could be written as

$$
\begin{aligned}
& c \cdot T_{2}(r, \varphi, z, t)=\lambda \int_{0}^{t} \frac{\partial^{2} T_{1}(r, \varphi, z, \tau)}{\partial r^{2}} d \tau+\lambda \frac{1}{r^{2}} \int_{0}^{t} \frac{\partial^{2} T_{1}(r, \varphi, z, \tau)}{\partial \varphi^{2}} d \tau+\lambda \int_{0}^{t} \\
& \frac{\partial^{2} T_{1}(r, \varphi, z, \tau)}{\partial z^{2}} d \tau+\int_{0}^{t} p(r, \varphi, z, \tau) d \tau-c \cdot \frac{\partial}{\partial r} \int_{0}^{t}\left\{v _ { r } ( r , \varphi , z , \tau ) \cdot \left[\alpha_{2 C}\right.\right. \\
& \left.\left.+C_{1}(r, \varphi, z, \tau)\right] \cdot\left[\alpha_{2 T}+T_{1}(r, \varphi, z, \tau)\right]\right\} d \tau+T_{r}-\frac{c}{r} \frac{\partial}{\partial \varphi} \int_{0}^{t}\left\{v _ { \varphi } ( r , \varphi , z , \tau ) \cdot \left[\alpha_{2 C}\right.\right.
\end{aligned}
$$




$$
\begin{gathered}
\left.\left.+C_{1}(r, \varphi, z, \tau)\right] \cdot\left[\alpha_{2 T}+T_{1}(r, \varphi, z, \tau)\right]\right] d \tau-c \cdot \frac{\partial}{\partial z} \int_{0}^{t}\left\{v_{r}(r, \varphi, z, \tau) \cdot\left[\alpha_{2 C}+C_{1}(r, \varphi, z, \tau)\right]\right. \\
\left.\cdot\left[\alpha_{2 T}+T_{1}(r, \varphi, z, \tau)\right]\right\} d \tau, \\
C_{2}(r, \varphi, z, t)=\frac{1}{r} \frac{\partial}{\partial r} \int_{0}^{t} r D \frac{\partial C_{1}(r, \varphi, z, \tau)}{\partial r} d \tau+\frac{1}{r^{2}} \frac{\partial}{\partial \varphi} \int_{0}^{t} D \frac{\partial C_{1}(r, \varphi, z, \tau)}{\partial \varphi} d \tau \\
+\frac{\partial}{\partial z} \int_{0}^{t} D \times \frac{\partial C_{1}(r, \varphi, z, \tau)}{\partial z} d \tau-\frac{1}{r} \frac{\partial}{\partial r}\left\{\int_{0}^{t} r\left[\alpha_{2 C}+C_{1}(r, \varphi, z, \tau)\right] \cdot v_{r}(r, \varphi, z, \tau) d \tau\right\} \\
-\frac{1}{r} \frac{\partial}{\partial \varphi} \int_{0}^{t} v_{\varphi}(r, \varphi, z, \tau) \cdot\left[\alpha_{2 C}+C_{1}(r, \varphi, z, \tau)\right] d \tau-\frac{\partial}{\partial z} \int_{0}^{t}\left[\alpha_{2 C}+C_{1}(r, \varphi, z, \tau)\right] \\
\cdot \nu_{z}(r, \varphi, z, \tau) d \tau+C_{0} \delta(z+L) . \quad(18)
\end{gathered}
$$

Average values of the second-order approximations of temperature and concentration of mixture $\alpha_{2 T}$ and $\alpha_{2 C}$ have been calculated by using the following standard relations:

$$
\begin{aligned}
& \alpha_{2 T}=\frac{1}{\pi \Theta R^{2} L} \int_{0}^{\Theta} \int_{0}^{R} r \int_{0}^{2 \pi} \int_{-L}^{L}\left(T_{2}-T_{1}\right) d z d \varphi d r d t, \\
& \alpha_{2 C}=\frac{1}{\pi \Theta R^{2} L} \int_{0}^{\Theta} \int_{0}^{R} r \int_{0}^{2 \pi} \int_{-L}^{L}\left(C_{2}-C_{1}\right) d z d \varphi d r d t .
\end{aligned}
$$

Substitution of the first- and the second-order approximations of temperature and concentration of mixture into relations (19) gives us possibility to obtain equations to determine required average values

$$
\begin{aligned}
& \alpha_{2 T}=\left(\frac{\lambda \sigma}{c \pi \Theta R L} \int_{0}^{\Theta}(\Theta-t) \int_{0}^{2 \pi} \int_{-L}^{L} T^{4}(R, \varphi, z, t) d z d \varphi d t-\int_{0}^{\Theta}(\Theta-t) \int_{0}^{2 \pi} \int_{-L}^{L} T_{1}\right. \\
& \times(R, \varphi, z, t) d z d \varphi d t \frac{\lambda}{c \pi \Theta R^{2} L}+\frac{\lambda}{c \pi \Theta R^{2} L} \int_{0}^{\Theta}(\Theta-t) \int_{0}^{2 \pi} \int_{-L}^{L} T_{1}(0, \varphi, z, t) d z d \varphi d t
\end{aligned}
$$




$$
\begin{aligned}
& -\int_{0}^{\Theta}(\Theta-t) \int_{0}^{2 \pi} \int_{-L}^{L} \nu_{r}(R, \varphi, z, t)\left\{\left[\alpha_{2 C}+C_{1}(R, \varphi, z, t)\right] T_{1}(R, \varphi, z, t)-\alpha_{1 T} \alpha_{1 C}\right\} \\
& \times d z d \varphi d t \frac{\lambda}{\pi \Theta R L}-\frac{1}{\pi \Theta R^{2} L} \int_{0}^{\Theta}(\Theta-t) \int_{0}^{R} \int_{0}^{2 \pi} \int_{-L}^{L} \nu_{r}(r, \varphi, z, \tau)\left\{T_{1}(r, \varphi, z, t)\right. \\
& \left.\times\left[\alpha_{2 C}+C_{1}(r, \varphi, z, t)\right]-\alpha_{1 T} \alpha_{1 C}\right\} d z d \varphi r d r d t-\frac{V_{0}}{\pi \Theta R^{2} L} \int_{0}^{\Theta}(\Theta-t) \int_{0}^{R} r \int_{0}^{2 \pi}\left[\left(\alpha_{2 C}+C_{0}\right)\right. \\
& \left.\cdot T_{1}(r, \varphi, L, t)-\alpha_{1 T} \alpha_{1 C}\right] d \varphi d r d t\left\{1+\frac{1}{\pi \Theta R L} \int_{0}^{\Theta} \int_{0}^{2 \pi} \int_{-L}^{L} v_{r}(R, \varphi, z, t)\left[\alpha_{2 C}\right.\right. \\
& \left.+C_{1}(R, \varphi, z, t)\right] d z d \varphi(\Theta-t) d t-\frac{1}{\pi \Theta R^{2} L} \int_{0}^{\Theta}(\Theta-t) \int_{0}^{R} r \int_{0}^{2 \pi} \int_{-L}^{L}\left[\alpha_{2 C}+C_{1}(r, \varphi, z, t)\right] \\
& \left.\times \nu_{r}(r, \varphi, z, \tau) d z d \varphi d r d t+2 V_{0}\left(\alpha_{2 C}+C_{0}\right) \frac{\Theta}{L}\right\}^{-1}, \\
& \alpha_{2 C}=\frac{1}{\pi \Theta R^{2} L} \int_{0}^{\Theta}(\Theta-t) \int_{0}^{R} r \int_{0}^{2 \pi} D\left[\left.\frac{\partial C_{1}(r, \varphi, z, \tau)}{\partial z}\right|_{z=L}-\left.\frac{\partial C_{1}(r, \varphi, z, \tau)}{\partial z}\right|_{z=-L}\right] \\
& \times d \varphi d r d t-\frac{1}{\pi \Theta R^{2} L} \int_{0}^{\Theta}(\Theta-t) \int_{0}^{2 \pi} \int_{-L}^{L}\left\{r\left[\alpha_{2 C}-\alpha_{1 C}+C_{1}(R, \varphi, z, \tau)\right] \cdot \nu_{r}(R, \varphi, z, \tau)\right\} \\
& \times d z d \varphi d t-\frac{V_{0}}{\pi \Theta R^{2} L} \int_{0}^{\Theta}(\Theta-t) \int_{0}^{R} r \int_{0}^{2 \pi}\left(\alpha_{2 C}-\alpha_{1 C}+C_{0}\right) d z d \varphi d r d t .
\end{aligned}
$$

\section{Discussion}

In this section, we will estimate the rate of growth of epitaxial layers. The required velocity is determined by the following relation [21]: $V_{g} \approx D C / \delta \rho$, where $D$ is the diffusion coefficient of the gas mixture, $C$ is 
the concentration of the gas mixture, $\delta$ is the thickness of the diffusion layer, and $\rho$ is the density of the gas mixture. The thickness of the diffusion layer can be determined by using the following relation [21]: $\delta \approx 1.6 \sqrt[3]{D \nu} \sqrt{\nu / \omega}$, where $\nu$ is the viscosity of the gas mixture, $\omega$ is the rotation frequency of the substrate holder. The Figure 2(a) shows the dependence of the considered growth rate on temperature. The Figure 2 shows the dependence of the considered growth rate on temperature for different values of the inclination angle of the substrate holder and the pressure in the reactor. It follows from this figure that an increase in the temperature of the substrate leads to an increase in the rate of growth of the material depositing on the substrate. The appearance of this effect, apparently, is due to the fact that as the temperature of the substrate increases, the decomposition of the reactant gases accelerates and the material of the epitaxial layer accelerates from the results of their decay. At the same time, an increase in the growth temperature leads to an increase in the contribution of natural convection and slowing down the growth of epitaxial layers. 




Figure 2(a). Dependence of growth speed on temperature of substrate $T$. Curve 1 corresponds to vertical reactor. Curve 2 corresponds to horizontal reactor. Curve 3 corresponds to average value of sloping angle (i.e., $\psi=45^{\circ}$ ) of keeper of substrate in comparison with direction of flow gazes.

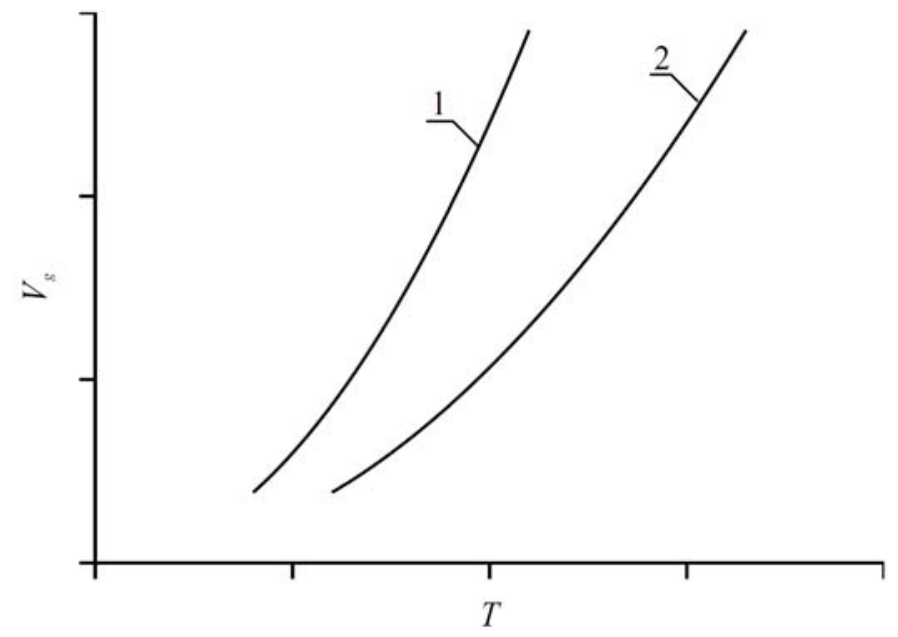

Figure 2(b). Dependence of growth speed on temperature of substrate $T$. Curve 1 corresponds to atmospheric pressure. Curve 2 corresponds to smaller pressure. 


\section{Conclusion}

In this paper, we estimate the rate of growth of epitaxial layers from the gas phase. We study its dependence on the amount of heating of the substrate. By using the previously proposed mass and heat transfer analysis, analytical dependencies of the considered velocity on the parameters were obtained.

\section{References}

[1] I. P. Stepanenko, Basis of Microelectronics, Soviet Radio, Moscow, 1980.

[2] V. G. Gusev and Yu. M. Gusev, Electronics, Higher School, Moscow, 1991.

[3] V. I. Lachin and N. S. Savelov, Electronics, Rostov-on-Don, Phoenix, 2001.

[4] A. A. Vorob'ev, V. V. Korablev and S. Yu. Karpov, The use of magnesium to dope gallium nitride obtained by molecular-beam epitaxy from activated nitrogen, Semiconductors 37(7) (2003), 838-842.

DOI: https://doi.org/10.1134/1.1592861

[5] L. M. Sorokin, N. V. Veselov, M. P. Shcheglov, A. E. Kalmykov, A. A. Sitnikova, N. A. Feoktistov, A. V. Osipov and S. A. Kukushkin, Electron-microscopic investigation of a $\mathrm{SiC} / \mathrm{Si}(111)$ structure obtained by solid phase epitaxy, Technical Physics Letters 34(11) (2008), 992-994.

DOI: https://doi.org/10.1134/S1063785008110278

[6] V. V. Lundin, A. V. Sakharov, E. E. Zavarin, M. A. Sinitsyn, A. E. Nikolaev, G. A. Mikhailovsky, P. N. Brunkov, V. V. Goncharov, B. Ya. Ber, D. Yu. Kazantsev and A. F. Tsatsulnikov, Effect of carrier gas and doping profile on the surface morphology of MOVPE grown heavily doped GaN:Mg layers, Semiconductors 43(7) (2009), 963-967.

DOI: https://doi.org/10.1134/S1063782609070276

[7] Y. E. Bravo-García, P. Rodríguez-Fragoso, J. G. Mendoza-Alvarez and G. Gonzalez de la Cruz, Growth and characterization of InAsSb layers on GaSb substrates by liquid phase epitaxy, Materials Science in Semiconductor Processing 40 (2015), 253-256.

$$
\text { DOI: https://doi.org/10.1016/j.mssp.2015.06.062 }
$$

[8] Y. Li, L. E. Antonuk, Y. El-Mohri, Q. Zhao, H. Du, A. Sawant and Yi Wang, Effects of $\mathrm{x}$-ray irradiation on polycrystalline silicon, thin-film transistors, Journal of Applied Physics 99(6) (2006); Article 064501.

DOI: https://doi.org/10.1063/1.2179149 
[9] A. Chakraborty, H. Xing, M. D. Craven, S. Keller, T. Mates, J. S. Speck, S. P. Den Baars and U. K. Mishra, Nonpolar $a$-plane $p$-type GaN and $p$ - $n$ Junction Diodes, Journal of Applied Physics 96(8) (2004); Article 4494.

DOI: https://doi.org/10.1063/1.1790065

[10] H. Taguchi, S. Miyake, A. Suzuki, S. Kamiyama and Y. Fujiwara, Evaluation of crystallinity of GaN epitaxial layer after wafer dicing, Materials Science in Semiconductor Processing 41 (2016), 89-91.

DOI: https://doi.org/10.1016/j.mssp.2015.07.083

[11] M. Mitsuhara, M. Ogasawara and H. Sugiura, Beryllium doping of InP during metalorganic molecular beam epitaxy using bismethylcyclopentadienyl-beryllium, Journal of Crystal Growth 183(1-2) (1998), 38-42.

DOI: https://doi.org/10.1016/S0022-0248(97)00336-9

[12] R. A. Talalaev, E. V. Yakovlev, S. Yu. Karpov and Yu. N. Makarov, On low temperature kinetic effects in metal-organic vapor phase epitaxy of III-V compounds, Journal of Crystal Growth 230(1-2) (2001), 232-238.

DOI: https://doi.org/10.1016/S0022-0248(01)01354-9

[13] H. S. Carslaw and J. C. Jaeger, Conduction of Heat in Solids, Clarendon Press, Oxford, 1964.

[14] Yu. D. Sokolov, About the definition of dynamic forces in the mine lifting, Applied Mechanics 1(1) (1955), 23-35.

[15] E. L. Pankratov and E. A. Bulaeva, On prognosis of epitaxy from gas phase process to improve properties of epitaxial layers, 3D Research 6(4) (2015); Article 40.

DOI: https://doi.org/10.1007/s13319-015-0073-4

[16] E. L. Pankratov and E. A. Bulaeva, On optimization of regimes of epitaxy from gas phase: Some analytical approaches to model physical processes in reactors for epitaxy from gas phase during growth films, Reviews in Theoretical Science 3(4) (2015), 365-398.

$$
\text { DOI: https://doi.org/10.1166/rits.2015.1041 }
$$

[17] E. L. Pankratov and E. A. Bulaeva, On optimization of growth from gas phase in a vertical reactor with account native convection to improve properties of films, Multidiscipline Modeling in Materials and Structures 12(4) (2016), 712-725.

\section{DOI: https://doi.org/10.1108/MMMS-02-2016-0008}

[18] E. L. Pankratov and E. A. Bulaeva, On development of analytical approaches to prognosis of epitaxial grows from gas phase to improve properties of films, Journal of Computational and Theoretical Nanoscience 14(7) (2017), 3556-3563.

DOI: https://doi.org/10.1166/jctn.2017.6787 
[19] E. L. Pankratov and E. A. Bulaeva, Modelling mass and heat transport during gas phase epitaxy in a reactor with rotating substrate: On possibility to improve of properties of films, International Journal of Thin Films Science and Technology 6(2) (2017), 53-59.

$$
\text { DOI: https://doi.org/10.18576/ijtfst/060201 }
$$

[20] G. A. Korn and T. M. Korn, Mathematical Handbook for Scientists and Engineers: Definitions, Theorems, and Formulas for Reference and Review, Second Edition, McGraw-Hill Book Company, New York, 1968.

[21] V. G. Levich, Physicochemical hydrodynamics, M. Наука, 1962. 